\title{
FIBROMA ODONTOGÉNICO CENTRAL: REPORTE DE CASO
}

\section{CENTRAL ODONTOGENIC FIBROMA: CASE REPORT}

\author{
Farfán Christian. ${ }^{1 *}$, Arce-Lazo Marco. ${ }^{2}$ \\ ${ }^{1}$ Residente de cuarto año de Cirugía Bucal y Maxilofacial - Hospital Regional Honorio Delgado -Universidad Nacional \\ Mayor de San Marcos.Perú. \\ ${ }^{2}$ Cirujano Maxilofacial - Hospital Regional Honorio Delgado de Arequipa.Perú. \\ *christianjr_88_@hotmail.com
}

\begin{abstract}
Resumen
El fibroma odontogénico es un tumor odontogénico raro de origen mesodérmico, que se origina del folículo dentario, ligamento periodontal y/o la papila dental. ${ }^{1}$ Diferentes estudios informaron una alta variabilidad en la tasa de incidencia como los comprendidos entre 3 y $23 \%$ de todos los tumores odontogénicos. ${ }^{2,3}$ Las características histopatológicas muestra las estructuras epiteliales odontogénicas que imitan a los observados en la biopsia de ameloblastoma y además fragmentos de estroma celular. Las características clínicas y radiológicas son similares a otros tumores odontogénicos y no odontogénicos.En el caso reportado, un paciente joven mostró un aumento de volumen a nivel de mucosa retromolar inferior que implica radiológicamente expansión de tabla ósea vestibular y palatina en ángulo mandibular derecho. Las características morfológicas, junto con hallazgos clínicos, imaginológicos e histopatológicos confirmaron que el tumor era un fibroma odontogénico central. Los tumores odontogénicos benignos raros pueden ser distinguidos de otras neoplasias odontogénicas / no odontogénicas y de tumores malignos a través de un diagnóstico diferencial histopatológico del cual depende el tratamiento a realizar.
\end{abstract}

Palabras clave: Tumor odontogénico, fibroma, diagnóstico diferencial.

\begin{abstract}
The odontogenic fibroma is a rare odontogenic tumor of mesodermal origin, originating from the dental follicle, periodontal ligament and/or the dental papilla. 1 Different studies reported a high variability in the incidence rate, ranging from $3 \%$ to 23\% of all odontogenic tumors. 2, 3 The histopathological features show the odontogenic epithelial structures that mimic those observed in ameloblastoma biopsy and also cell stroma fragments. The clinical and radiological features are similar to other odontogenic and non odontogenic tumors. In the case reported, a young patient showed an increased volume at the level of inferior retromolar mucosa that radiologically involves expansion of the buccal and palatal bone table at the right mandibular angle. Morphological characteristics, together with clinical, imaging and histopathological findings, confirmed that the tumor was a central odontogenic fibroma. Rare benign odontogenic tumors can be distinguished from other odontogenic/nonodontogenic neoplasms and malignant tumors through a differential histopathological diagnosis on which the treatment to be performed depends.
\end{abstract}

Key words: Odontogenic tumor, fibroma, differential diagnosis.

\section{INTRODUCCIÓN}

El fibroma odontogénico es un tumor raro de origen odontogénico, con porcentajes variables de incidencia, ${ }^{2,3}$ considerado por la Organización Mundial de la Salud (OMS) como una neoplasia odontogénica benigna derivada de tejido odontogénico mesenquimal. ${ }^{4}, 5$ La lesión se produce con mayor frecuencia en la mandíbula aunque se han descrito casos en el maxilar superior. ${ }^{6}$ Se ha reportado en pacientes de edades comprendidas entre 11 y 80 años con una edad media de 34 años. Daley et al. informó en una revisión bibliográfica de un ligero predominio femenino. ${ }^{7}$ La lesión crece lentamente, de una manera asintomática. En las radiografías aparece como una imagen radiolúcida bien definida, o como una lesión multilocular con frecuencia asociada con los dientes que no han erupcionado o desplazados, contribuyendo al desplazamiento de tablas óseas. Histopatológicamente se caracteriza por una cantidad variable de epitelio odontogénico de aspecto inactivo incrustado en un estroma fibroso denso. Wesley et al. tuvo el mérito de aclarar la naturaleza del tumor, diferenciándolo de otras entidades patológicas como mixoma odontogénico, folículo dentario hiperplásico y otras lesiones fibroóseas. ${ }^{1}$ Gardner ha identificado tres variantes histológicas de Fibroma Odontogénico: hiperplasia fibrosa, el tipo simple (pobre en epitelio) y el tipo complejo (rico en epitelio). ${ }^{8}$ Topográficamente se pueden distinguir dos variantes: Tipo extraóseo o periférico y un tipo intraóseo o 
central. ${ }^{4,5}$ La Fig 1 resume las diferentes clasificaciones:

En este artículo presentamos las características clínicas, imagenelógicas, morfológicas e histopatológicas de un caso de Fibroma Odontogénico Central, con el objetivo de reforzar el concepto de un enfoque correcto necesario para el tratamiento de tumores odontogénicos.

\section{PRESENTACIÓN DEL CASO}

Paciente varón de 24 años con un tiempo de enfermedad de 2 años, es referido por un odontólogo general, ya que al realizar exodoncia de pieza dentaria 4.8 , refirió como hallazgo una imagen radiolúcida compatible con quiste mandibular. Al exámen extraoral se evidenció presencia de asimetría facial por una aumento de volumen a nivel submandibular derecho, indoloro a la palpación, no crepitación, a la evaluación intraoral se evidencia un aumento de volumen a nivel de tabla vestibular y lingual, distal a pieza dentaria 4.7 que no presenta movilidad y leve dolor a la percusión. La radiografía panorámica anterior a exodoncia y actual evidencia una imagen radiolucida con bordes definidos en distal de pieza dentaria 4.7 que a aumentado en dimensión y ocasionó el desplazamiento de la pieza 4.8 antes de su exodoncia. Al realizar la evaluación tomografía, evidenciamos un imagen hipodensa con borde definidos a nivel distal de pieza 4.7 a nivel de ángulo mandibular derecho que no involucra hueso basal. Tanto la evaluación clínica como imagenologica nos lleva al posible diagnóstico de un tumor odontogénico benigno. Véase en la Fig 1.

Se realiza biopsia incisional obteniendo muestra que mide 1 x 0.7 × $0.8 \mathrm{~cm}$ fijada en formol al $10 \%$, a la evaluación microscópica se evidencia proliferación de fibroblastos y fibras de colageno distribuidas de manera irregular, se aprecia pequeñas islas de epitelio odontogénico ,algunas de estas células muestran ligera atipia y moderada vascularización ,en las superficie se aprecian trabeculas de hueso reactivo compatible con Fibroma Odontogénico Central. Se realiza remoción de tumoración en sala de operaciones. El paciente evoluciona favorablemente y se realiza controles posteriores donde no se evidencia recidiva de tumoración. Véase en la Fig 2.

\section{DISCUSIÓN}

El fibroma odontogénico es una lesión rara origen de origen odontogénico ectomesenquimal que presenta un crecimiento lento .La Organización Mundial de la Salud (OMS) la ha definido como una neoplasia odontogénica benigna de origen fibroblástico caracterizado por tejido fibroso de colágeno maduro relativo con o sin cantidades variables de epitelio odontogénico de aspecto inactivo integrado con potencial de ocurrir ya sea en una ubicación central o extraósea. ${ }^{5}$ Las diferencias y similitudes clínico-patológicas entre fibroma odontogénico central y fibroma odontogénico periférico se muestran a continuación:

\subsection{El fibroma odontogénico periférico.}

Clínicamente se presenta como una masa firme sésil de un color similar al del tejido conectivo que rodea y evoluciona como una lesión no ulcerada, de crecimiento lento, asintomática, exofítica, elevada, unida a la encía y cubierta por una mucosa de aspecto normal, clínicamente indistinguible de otras lesiones gingivales fibrosas comunes. ${ }^{16}$ Por lo general, se encuentra en la encía vestibular de la mandíbula, como una lesión solitaria que se origina principalmente a nivel del incisivo, canino y premolar. No implica el hueso subyacente y rara vez puede causar desplazamiento de los dientes. En raras ocasiones, son multifocales o difusas. También se ha informado asociación con lesiones oculares y de la piel. ${ }^{17,18}$ Histológicamente, es un tumor no encapsulado se caracteriza por la mezcla de tejido conectivo y las islas de epitelio odontogénico y la dentina displásica asociada, calcificaciones y trabéculas osteoides. ${ }^{19}$ Entre los subtipos histológicos existe la variante de células granulares. ${ }^{20}$ y la variante de células escamosas. ${ }^{13} \mathrm{El}$ tratamiento consiste en la escisión local y el pronóstico es excelente. ${ }^{21}$ La tasa de recurrencia es significativa, variando desde muy bajo hasta un máximo de $38,9 \%$. ${ }^{22}$ Por la posibilidad de recurrencia, ${ }^{7,23}$ la evaluación radiográfica, histopatológica y citológica es fundamental y el seguimiento es obligatorio.

\subsection{El fibroma odontogénico central.}

Esta lesión aparece como una expansión asintomática de la placa cortical de la mandíbula o el maxilar con la misma frecuencia. Generalmente la lesión provoca el aumento de volumen en la mandíbula. La lesión puede evolucionar a partir de un germen dental (papila dental o folículo) o de la membrana periodontal, y por lo tanto es invariablemente relacionada con la porción coronal o radicular de los dientes. Cabe señalar que el sitio más habitual de presentación en la mandíbula es la zona posterior, mientras que en el maxilar superior es la región anterior. Con más frecuencia en el grupo etario entre los 20 a 45 años, con predilección por las mujeres. Radiológicamente presenta imágenes radiolúcidas uni o multiloculares con bordes bien definidos. En algunos casos raros, podría presentar una imagen mixta radiolúcida y radiopaca con los bordes no definidos. La reabsorción radicular y el desplazamiento se han reportado en los casos de lesiones más graves. Histopatológicamente muestra características similares al Fibroma odontogénico periférico, que consiste en tejido conectivo fibroso de colágeno que contiene cantidades variables de epitelio odontogénico, en algún momento asociados a un gran componente celular granulomatoso. ${ }^{24}$ Responde bien a la enucleación quirúrgica o curetaje. La recurrencia es relativamente poco común .Se recomienda el seguimiento estricto a largo plazo. La opinión actual se refiere al fibroma odontogénico (tanto central como periférico) como una entidad distinta con los patrones clínico e histopatológico que lo diferencian de lesiones inflamatorias / reactivas o traumáticas, tales como tejido hiperplásico fibroso reactivo o folículo 


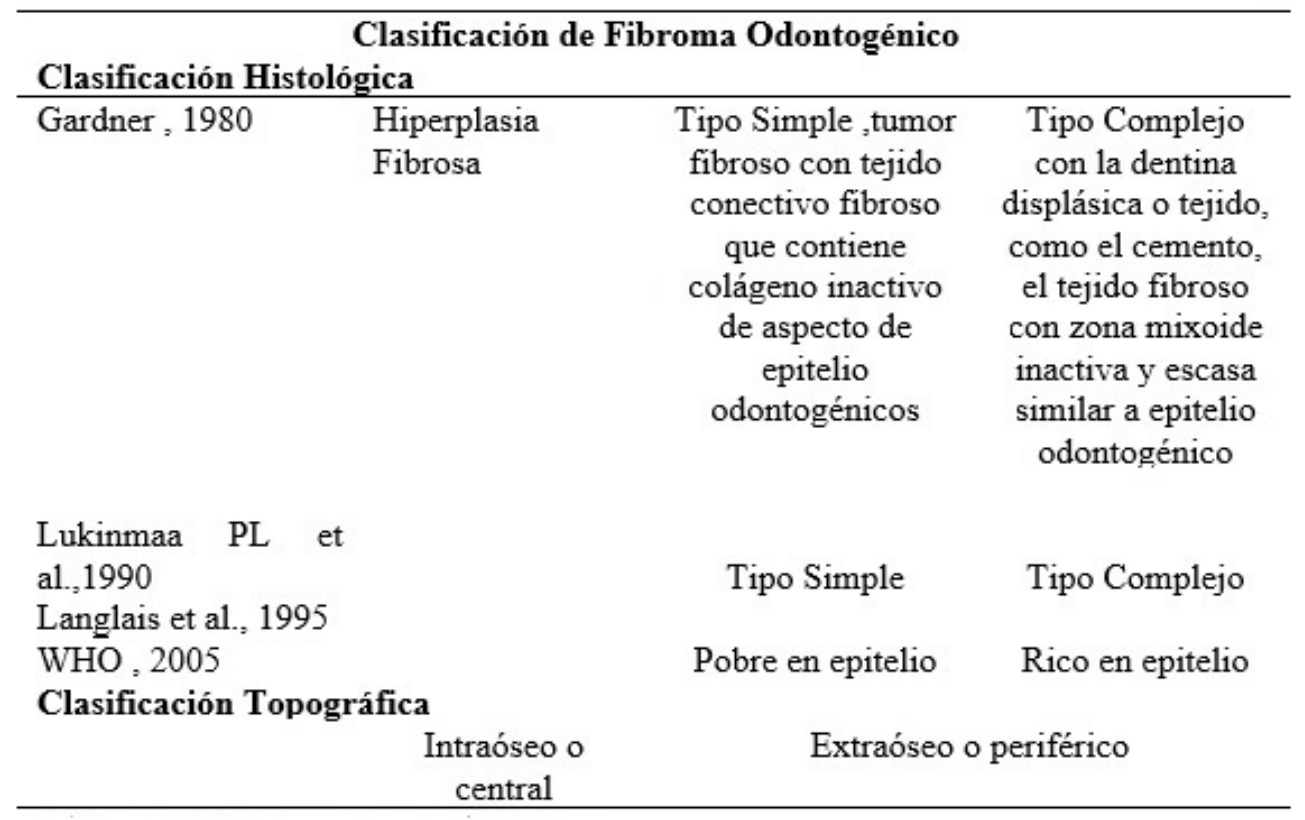

Tabla 1. Clasificación del fibroma Odontogénico

dental hiperplásico y quiste óseo traumático, sino también de numerosas otras lesiones como el fibroma, fibroma de células gigantes, fibroma osificante periférico, el fibroma desmoplásico, ameloblastoma, el fibroma ameloblástico, el carcinoma ameloblástico, tumor odontogénico calcificante quístico,tumor odontogénico epitelial calcificante, queratoquiste odontogénico, granuloma central de células gigantes, el granuloma periférico de células gigantes, el granuloma piógeno, mixoma odontogénico. De todos modos, ya que las características clínicas, histológicas y radiológicas de fibroma odontogénico a menudo no son muy claras, un enfoque multidisciplinario de diagnóstico clínico debe realizarse. En general, aunque el Fibroma odontogénico central surge en un amplio rango de edad con una edad media de 34 años, con una ligera predilección por las mujeres, en el caso que aquí se presenta, el paciente era un joven de 24 años de edad. Desde un punto de vista clínico el paciente joven mostró un aumento de volumen al inicio asociado a una pieza dentaria incluida, que podría haber sido interpretado como una forma de lesión reactiva o también como quiste odontogénico. La evaluación imagenelogica reveló el origen central real de la lesión, que se caracteriza por una localización del hueso en el margen superior del ángulo derecho de la mandíbula y limitada por un borde esclerótico radiopaco. Una aspiración con aguja fina, cuando sea posible, podría ser una herramienta de diagnóstico válido para realizar un diagnóstico diferencial citológico de otras lesiones no neoplásicas, benignas y malignas de la mandíbula. En nuestro caso, la biopsia guió el manejo del caso clínico, que luego fue confirmada por la evaluación histopatológica de la tumoración resecada. Generalmente, un diagnóstico de tumor odontogénico se puede hacer fácilmente por la presencia de epitelio odontogénico; sin embargo, en los casos en que las estructuras epiteliales odontogénicos están ausentes, esta simple conclusión no es posible, pero es necesario tener en cuenta una gran cantidad de posibilidades, en términos de diagnóstico diferencial.

\section{CONCLUSIÓN}

Un diagnóstico inicial incorrecto puede determinar un retraso grave y en caso de que la lesión tenga un tiempo de enfermedad prolongado puede llegar a erosionar el hueso circundante. En consecuencia, también la eficacia de un tratamiento adecuado podría ser excluida. Por lo tanto, este reporte de caso pone de relieve la importancia de realizar los exámenes clínicos e imaginologicos pertinentes y periódicos del paciente, subraya la importancia de realizar una biopsia adecuada para un examen histopatológico cuidadosa de las lesiones fibrosas de la mandíbula y de un diagnóstico diferencial adecuado entre los diversos tipos de tumores odontogénicos y no odontogénicos.

\section{CONSENTIMIENTO}

El consentimiento informado se obtuvo del paciente así como los familiares del paciente para la publicación de este caso y las imágenes adjuntas. Una copia de la autorización escrita está disponible para su revisión por el Editor en Jefe de la revista bajo petición. 


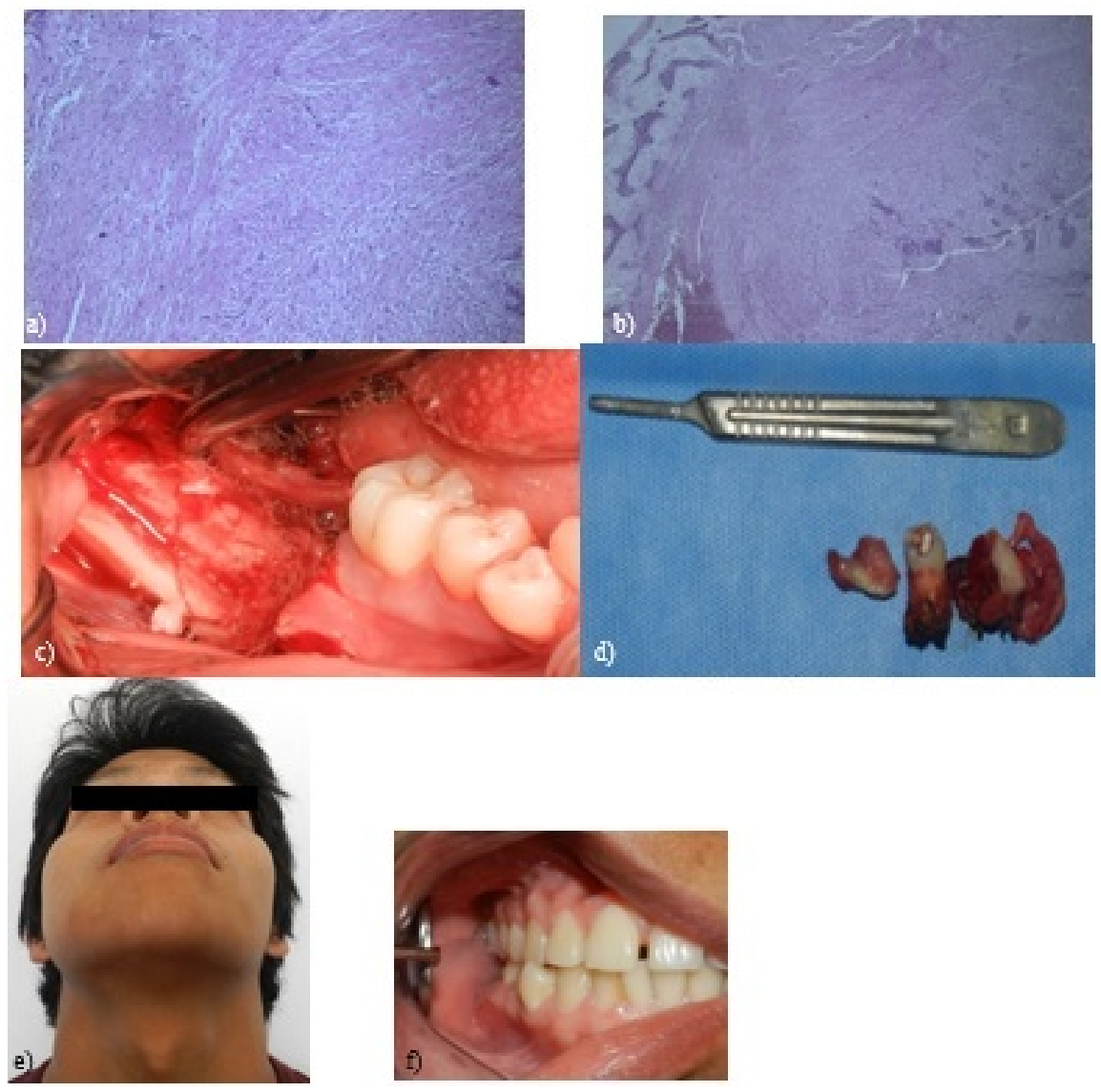

Fig. 1. a)Imagen histopatologica que muestra la proliferación de fibroblastos y fibras de colageno b)Imagen a mayor aumento que evidencia fibroblastos polimorofos y ligera atipia celular c) Resección quirurgica de tumoración d)Pieza quirúrgica que incluye pieza dentaria 4.7 e) Imagen extraoral de control f)Imagen intraoral que muestra la total evolucion y recuperacion de la herida operatoria g) y h)

\section{APROBACIÓN ÉTICA}

Las fuentes de financiación declaramos que no ha habido un importante apoyo financiero para este trabajo que podría haber influido en el resultado.

\section{DECLARACIÓN DE CONFLICTO DE INTERÉS}

Los autores desean confirmar que no existen conflictos de interés conocidos asociados con esta publicación y no ha habido un importante apoyo financiero para este trabajo que podría haber influido en el resultado. Los autores confirman que el manuscrito ha sido leído y aprobado por todos los autores mencionados y que no hay otras personas que cumplían los criterios de autoría, pero no figuran. 

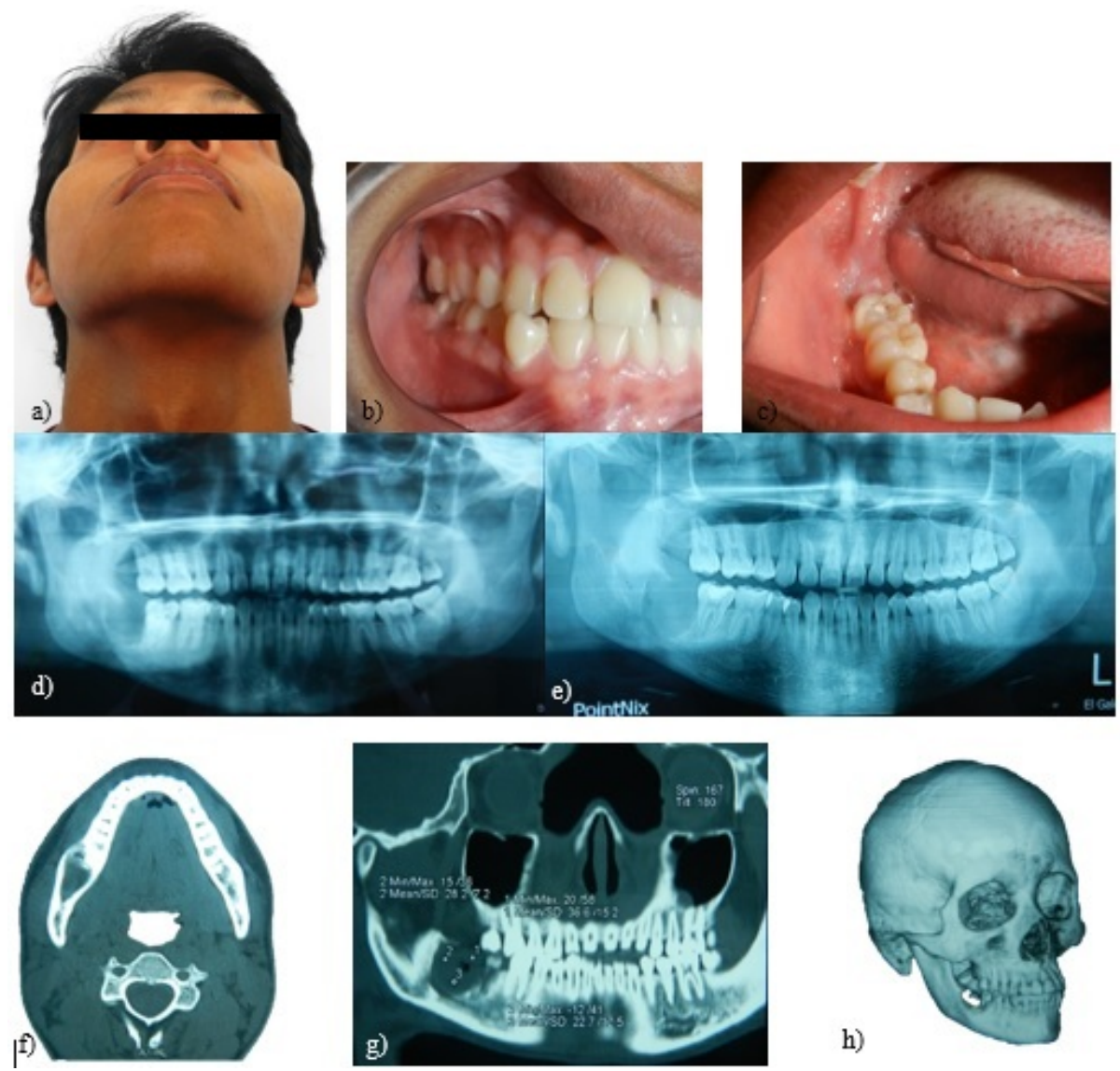

h)

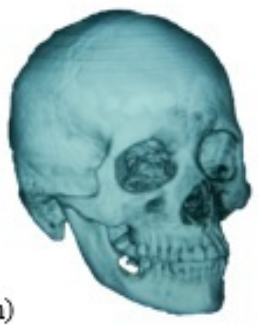

Fig. 2. : a)Examen extraoral b) y c) Imagen Intraoral que evidencia el desplazamiento de la cortical vestibular y lingual d)Rx panorámica 2014 donde de evidencia imagen radiolucida a distal de pieza 4.7 que ah desplazado pieza 4.8 e) Rx Panorámica 2016 muestra aumento de la dimensión de la imagen radiolucida f) g) y h) Evaluación tomografica que muestra imagen hipondesa con bordes definidos a nivel de ángulo mandibular derecho con compromiso óseo sin extenderse a basal mandibular

\section{Referencias}

1 Wesley R, Wysocki G, Mintz S. The central odontogenicfibroma clinical and morphological studies Oral Surg., 40 (1975), p. 235

2 Bhaskar S. Synopsis of Oral Pathology (fifth ed.)The CV Mosby Company, St. Louis (1977), pp. 259-261x

3 LeDoussal V, Mahe E, Herbert H. Los fibromyxomes odontogenes Arch. Anat. Cytol. Pathol, 23 (1981), pp. 325-331

4 Philipsen H, Reichart P. Classification of odontogenic tumours. A historical review J. Oral Pathol. Med., 2006, pp. $525-529$

5 Philipsen H.P, Reichart P.A, Sciubba J.J, van der Waal I. Odontogenic fibroma Pág. 315 León Barnes, John W. Eveson, Peter Reichart, David Sidransky (Eds.), World Health Organization Classification of Tumours. Pathology and Genetics of Head and Neck Tumours, IARC Press Lyon (2005)

6 Dahl E.C, Wolfson S.H, Haugen Central J.C. odontogenic fibroma: a review of literature and report of a case J. Oral Surg., 39 (1981), p. 120
7 Daley T.D, Wysocki G.P. Peripheral odontogenic fibroma Oral Surg. Oral Med. Oral Pathol., 78 (1994), pp. 329-336

8 Gardner D.G. The central odontogenic fibroma: an attempt at clasification Oral Surg., 50 (1980), p. 425

9 Lukinmaa P.L, Hietanee J, Anttinen J, Ahonen P. Continuous enlarged dental follicles with histologic features resembling the WHO type of odontogenic fibroma Oral Surg. Oral Med. Oral Pathol., 70 (1990), pp. 313-317

10 Langlais R.P, Langland O.E, Nortje C.J. Multilocular radiolucencies Diagnostic Imaging of the Jaws, Williams \& Wilkins, Baltimore (1995), pp. 370-376

11 Baden E, Moskow B.S, Moskow R. Odontogenic gingival epithelial hamartoma J. Oral Surg., 26 (1968), pp. 702-714

12 Baden E, Moskow B.S, Moskow R. Odontogenic gingival epithelial hamartoma J. Oral Surg., 26 (1968), pp. 702-714

13 Sciubba J, Zola M. Odontogenic epithelial hamartoma Oral Surg. Oral Med. Oral Pathol., 45 (1978), pp. 261-265

14 Gardner D. The peripheral odontogenic fibroma: an attempt at clarification Oral Surg. Oral Med. Pathol., 54 (1982), pp. $40-48$ 
15 Buchner A, Merrell P, Carpenter W. Relative frequency of central odontogenic tumours: a study of 1088 cases from northern California and comparison to studies from other parts of the world J. Oral Maxillofac. Surg., 64 (2006), pp. $1343-1352$

16 Buchner A, Ficarra G, Hansen L. Peripheral odontogenic fibroma Oral Surg. Oral Med. Oral Pathol., 64 (4) (1987 Oct), pp. 432-438

17 Neville B, Damm D, Allen C, Bouquot J. Oral and Maxillofacial Pathology (third ed.)WB Saunders, Philadelphia (2009) pp. 729-9

18 Weber A, P. van Heerden W, Ligthelm A, Raubenheimer E. Diffuse peripheral odontogenic fibroma: report of 3 cases J. Oral Pathol. Med., 21 (1992), pp. 82-84

19 Kenney J, Kaugars G, Abbey L. Comparison between the peripheral ossifying fibroma and peripheral odontogenic fibroma J. Oral Maxillofac. Surg., 47 (4) (1989 Apr), pp. 378-382

20 Lownie J, Altini M, Shear M. Granular cell peripheral odontogenic fibroma J. Oral Pathol. Med., 5 (1976), pp. 295-304

21 Baiju C. Rohatgi Sumidha Peripheral odontogenic fibroma: a case report and review J. Indian Soc. Periodontol., 15 (3) (2011 Jul-Sep), pp. 273-275

22 Michaelides P. Recurrent peripheral odontogenic fibroma of attached gingiva: a case report J. Periodontol., 63 (1992), pp. 645-647

23 Armas J, Hunter K, Jenkins W. Odontogenic fibroma: an unusual presentation J. Oral Maxillofac. Pathol., 12 (2008), pp. $68-71$

24 Allen C, Hammond H, Stimson P. Central odontogenic fibroma, WHO type. A report of three cases with an unusual associated giant cell reaction Oral Surg. Oral Med. Oral Pathol., 73 (1) (1992 Jan), pp. 62-66 fibroma of the mandible Contemp. Clin. Dent., 3 (2) (2012 Apr-Jun), pp. 230-233

Recibido: 02 de Febrero de 2016

Aceptado: 12 de Febrero de 2016 